\title{
Retour sur la session d'automne des Chambres fédérales
}

La médecine a été le thème le plus important lors de la dernière session des Chambres. Pas de panique: il n'y a eu aucune révolution! Ce n'est pas la médecine politique qui a été au centre, mais la médecine clinique: la vraie médecine! Celle qui a permis de sauver la vie du conseiller fédéral Hans-Rudolf Merz, qui a subi un arrêt cardiocirculatoire. La médecine a gagné la guerre des spéculations politiques: l'armée des candidats à la succession peut rentrer chez elle. Monsieur Merz - grâce à notre médecine moderne - va bien et nous en sommes heureux: nous lui souhaitons une bonne guérison!

Les thèmes centraux de la session étaient la politique de sécurité et l'armée. Le programme d'armement 2008 a été refusé au Conseil national par une étrange coalition: gauche et droite contre l'armée (mais pour des raisons fort différentes!). Un débat urgent a aussi été dédié aux prix croissants du courant électrique.

Sur le front de la santé quatre thèmes ont été débattus: la protection contre le tabagisme, les médicaments (prix et importations parallèles), la médecine complémentaire et l'article constitutionnel sur la recherche sur l'être humain.

1. La protection du tabagisme s'est conclue avec l'approbation d'une loi qui interdit en Suisse la fumée dans tous les lieux publics, y compris restaurants, bars, discothèques, etc. Mais dans le souci de sauver les petits bistros de campagne et de montagne, la loi accorde aux propriétaires la liberté de déclarer «entièrement fumeur» les bistros dont la surface accessible au public est inférieure à $80 \mathrm{~m}^{2}$. Des querelles juridiques sur la mensuration de ces surfaces sont d'ores et déjà annoncées! Les cantons ont quand même le droit d'édicter des lois plus restrictives (p. ex. Tessin) [04.476].

2. Pour les médicaments, deux dossiers étaient en discussion: une modification de la LAMal, appelée de manière un peu mystérieuse «managed care» [04.062], et la loi sur les brevets, qui touche la question des importations parallèles des médicaments [08.010].

La modification de la LAMal avait surtout pour but d'ancrer dans la loi des règles qui ont jusqu'ici été édictées par le Conseil fédéral dans ses ordonnances (inclusion et exclusion d'un médicament de la liste des spécialités, contrôle du prix du médicament, critères d'économicité, etc.). Ce faisant, le Parlement enlève un peu de pouvoir à l'Exécutif pour le gérer lui- même ... par exemple parce qu'il estime pouvoir le faire mieux. Cette modification de la loi a duré quatre ans et le Parlement n'a pas réussi à trouver une solution acceptable pour la majorité. Quatre ans de travail à la poubelle: la modification de loi a été rejetée. Tout reste comme avant.

La question des importations parallèles a beaucoup fait parler d'elle: c'est un mot magique, qui cache une réalité fort complexe: régime d'épuisement national, européen, international ... les deux Chambres sont toujours en désaccord, le ping-pong n'est pas fini. C'est le retour au Conseil des Etats.

3. Pour la médecine complémentaire [06.066] le travail est conclu: le Parlement a adopté un contreprojet à l'initiative populaire, qui - selon ses auteurs - devrait ainsi être retirée. La population suisse devra prochainement décider si elle veut inscrire dans la Constitution le nouvel article 118a: «La Confédération et les cantons pourvoient, dans les limites de leurs compétences respectives, à la prise en compte des médecines complémentaires.» Une fois cette phrase inscrite dans la Constitution, il faudra lui donner suite au niveau des lois: le chemin est encore long. L'acceptation de cet article par le peuple ne signifie en tout cas pas la réintroduction immédiate des cinq prestations de médecine complémentaire biffées en 2005: ceci reste une compétence du chef du DFI.

4. L'article constitutionnel concernant la recherche sur l'être humain [07.072] est né dans le but de transférer cette compétence des cantons à la Confédération et d'harmoniser la matière. Il a été analysé en première lecture par le Conseil national, qui a décidé de ne garder que le premier alinéa des trois proposés par le Conseil fédéral. C'est maintenant le tour du Conseil des Etats.

Voici donc un bref aperçu de cette session d'automne. Vous désirez en savoir davantage? Mettez les petits numéros entre parenthèse dans la case «recherche» de la page www.parlament.ch! Bon voyage!

Dr Ignazio Cassis, $\mathrm{MPH}$, vice-président de la FMH et conseiller national 